\title{
Study of exact solutions of nonlinear heat equations
}

\author{
A. EBADIAN and P. DARANIA \\ Department of Mathematics, Faculty of Science, Urmia University, P.O. Box 165, Urmia, Iran \\ E-mails: a.ebadian@mail.urmia.ac.ir/p.darania@mail.urmia.ac.ir
}

\begin{abstract}
One of the ways that energy transports in fluid is electron thermal conduction. The aim of Inertial Confinement Fusion (ICF) is to show that the thermal conductivity is strongly dependent on temperature and the equation of heat condition is a nonlinear equation. In this article, we analyze the exact solutions of the nonlinear equation of heat conduction problem with variable transfer coefficients which is the problem of ICF.
\end{abstract}

Mathematical subject classification: 35K05, 35A20.

Key words: discrete group transformations, heat equation, Emden-Fowler equations.

\section{Introduction}

Inertial confinement fusion is a process where nuclear fusion reactions are initiated by heating and compressing a target - a pellet that most often contains deuterium and tritium by the use of intense laser or ion beams. The beams explosively detonate the outer layers of the target, accelerating the remaining target layers inward and sending a shock wave into the center. If the shock wave is powerful enough and if high enough density at the center is achieved some of the fuel will be heated enough to cause fusion reactions, releasing energy. In a target which has been heated and compressed to the point of thermonuclear ignition, energy can then heat surrounding fuel to cause it to fuse as well, creating a chain reaction that burns the fuel load, potentially releasing tremendous amounts of energy. Theoretically, if the reaction completes with perfect efficiency (though this is a practically impossible feat), a small amount of fuel about the size of a 
pinhead, or around 10 milligrams, is capable of releasing the energy equivalent to burning a barrel of oil (that is, chemically combining its hydrocarbon molecules with oxygen).

Fusion reactions combine lightweight atoms, such as hydrogen, together to form larger ones. Generally the reactions take place at such high temperatures that the atoms have been ionized, their electrons stripped off by the heat; thus, fusion is typically described in terms of "nuclei" instead of "atoms".

Fusion reactions on a scale useful for energy production require a very large amount of energy to initiate in order to overcome the so-called Coulomb barrier or fusion barrier energy. Since the positively-charged nuclei are naturally repelling each other, this repulsive force must be overcome by providing some form of external energy. When this occurs, however, the reaction is a rather energetic one. Generally less energy will be needed to cause lighter nuclei to fuse, and when they do, more energy will be released. As the mass of the nuclei increase, there is a point where the reaction no longer gives off net energy - the energy needed to overcome the energy barrier is greater than the energy released in the resulting fusion reaction. This point occurs when iron nuclei are formed and is the cause of death in some massive stars. This phenomenon plays no role in laboratory induced fusion however, since the energy and temperature required to form iron nuclei are very large. Fusion of heavy nuclei is possible using particle accelerators and is the method used to form very heavy transuranic elements such as Roentgenium for instance, though the method of achieving this fusion of heavy elements is far removed from the methods used in large scale fusion reactions which are desired in tokamak or ICF fusion reactors.

The key to practical fusion power is to select a fuel that requires the minimum amount of energy to start, that is, the lowest barrier energy. The best fuel from this standpoint is a one to one mix of deuterium and tritium; both are heavy isotopes of hydrogen. The D-T (Deuterium and Tritium) mix has a low barrier because of its high ratio of neutrons to protons. The presence of neutral neutrons in the nuclei helps pull them together via the strong force; while the presence of positively charged protons pushes the nuclei apart via Coloumbic forces (the electromagnetic force). Tritium has one of the highest ratios of neutrons to protons of any element - two neutrons and one proton. Adding protons or 
removing neutrons increases the energy barrier.

In order to create the required conditions, the fuel must be heated to tens of millions of degrees, and/or compressed to immense pressures. The temperature and pressure required for any particular fuel to fuse is known as the Lawson criterion. These conditions have been known since the 1950s when the first H-bombs were built.

ICF experiments started in earnest in the mid-1970s, when lasers of the required power were first designed. This was long after the successful design of magnetic confinement fusion systems, and even the particularly successful tokamak design that was introduced in the early 1970s. Nevertheless, high funding for fusion research stimulated by the multiple energy crises during the 1970's produced rapid gains in performance, and inertial designs were soon reaching the same sort of "below breakeven" conditions of the best magnetic systems.

One of the earliest serious attempts at an ICF design was Shiva, a 20-armed neodymium laser system built at the Lawrence Livermore National Laboratory (LLNL) that started operation in 1978. Shiva was a "proof of concept" design, followed by the NOVA design with 10 times the power. Funding for fusion research was severely constrained in the 80's, but NOVA nevertheless successfully gathered enough information for a next generation machine whose goal was ignition. Although net energy can be released even without ignition (the breakeven point), ignition is considered necessary for a practical power system.

In [1] and [2], we solved some classes of nonlinear Volterra integral equations and Emden-Fowler equation, by introducing the Discrete Group Transformations ( $D G T$ ) and $R F$-pair operations, which thus fur, could not be integrated using the classical method. Also in [3], we analyze relation between the Emden-Fowler equations and the nonlinear heat conduction problems with variable transfer coefficients by using the discrete group method. In this article, we analyze the exact solutions of the nonlinear equation of heat conduction problem with variable transfer coefficients which is the problem of ICF. The exact solutions of classical Emden-Fowler equation, by means of appropriate transformations of discrete group analysis is transformed into the exact solution of nonlinear applied problem of ICF. This approach is effective to find a new integrable equations which thus far, could not be integrated using the classical methods. 
This approach shows that, under the discrete group transformation, the solution of transformed equations can be converted into the solution of the reference equation $[1,2,5,6]$.

\section{Preliminaries}

It is well known that the theory of differential equations takes a central place among possible instruments for the modeling of different processes and phenomena. Generalized Emden-Fowler equations arise in many different fields of applied sciences such as fluid mechanics [5], chemical physics [8] and physics of plasma [9].

In the present paper we consider the set $\mathcal{D}$ of ordinary differential equations, which is referred to as a class of equations, whose members $D(x, y, a) \in \mathcal{D}$ are uniquely defined by a vector $a$ of parameters. The Discrete Group Methods $(D G M)$ based on the new fundamental mathematical theory is fully developed in $[5,6,10]$.

All the existing methods of exact solution of ordinary differential equations can be conditionally divided into two groups:

A) a search for transformation of the original ordinary differential equations in class $\mathcal{D}$ to some other class of ordinary differential equations $\mathcal{D}_{1}$, which belongs to one of standard classes of $O D E$ having known solutions.

B) a search for transformation leaving original ordinary differential equation in $\mathcal{D}$ invariant, i.e. transformation into "itself", that gives independent information about the solution.

The $D G M$ does not operate with a single equation as in applications of Lie method [5], but operates with a class of equations $\mathcal{D}$, depending on a vector $a$ of parameters, containing the investigated equation, but contrary to the approach $\mathbf{A}$ one consider the transformations of the given class $\mathcal{D}$ which are closed in itself on a chosen class of $O D E$.

Definition 2.1. The class of generalized Emden-Fowler equations is written as:

$$
y_{x x}^{\prime \prime}=A x^{n} y^{m}\left(y_{x}^{\prime}\right)^{l}, \quad a=(n, m, l),
$$


where the three-dimensional parameter vector $a=(n, m, l) \in \mathbb{R}^{3}$, and the parameter $A$ is insignificant. The parameter subspace $(n, m, 0)$ defines the set of classical Emden-Fowler equations

$$
y_{x x}^{\prime \prime}=A x^{n} y^{m} .
$$

Let $\mathcal{D}$ be a class of $O D E$ and

$$
D(x, y, a)=0,
$$

be an equation in this class, where $a$ is a vector parameter. We shall seek the transformations $g_{i}$ that are closed in the class (2), i.e., they change only the vector $a$

$$
g_{i}: D(x, y, a) \rightarrow D\left(t, u, b_{i}\right)
$$

if each $g_{i}$ has an inverse, then the collection $\left\{g_{i}\right\}$ defines a Discrete Transformation Groups (DGT) on the class (2).

Definition 2.2. An RF-Pair is an operation of consecutive raising and lowering the order of equation.

Now, we define the following R-operations and $F$-operations:

i) Termwise m-fold differentiation of the original equation, type $R D^{m}$.

ii) Termwise one or two-fold differentiation of original equation with respect to the independent variable, type accordingly $R X$ or $R X^{2}$.

iii) The equation is an exact derivative of the $m$ th order: termwise integration $m$ times, type $F I^{m}$.

iv) The equation is autonomous, i.e., it does not conclude an independent variable in an explicit form, type $F X$

$$
F X: y_{x}^{\prime}=u(y), \quad y_{x x}^{\prime \prime}=u u_{y}^{\prime} .
$$

v) The equation is homogeneous in the extended sense, type $F U$ : the transformation $x=e^{t}, y=u e^{k t}$, with an appropriate choice of $k$, leads to an autonomous form followed by a transformation $F X$.

If an $R(F)$-operating $R Z^{m}\left(F Z^{m}\right)$ is inverted, it is denoted by $R Z^{-m}\left(F Z^{-m}\right)$. 
Definition 2.3. Abel equation of the second kind, which is applied in many problems of mechanics, physics and other sciences is written as (see [5] for details):

$$
y y_{x}^{\prime}=F_{1}(x) y+F_{0}(y) .
$$

In literature, there are two methods for searching discrete group transformations, namely point transformations and Bäcklund transformations:

\subsection{Point transformations}

In the class of point transformations:

$$
y=f(t, u), \quad x=g(t, u), \quad J=f_{u} g_{t}-g_{u} f_{t} \neq 0,
$$

two methods are effectively applicable: the direct method and the method based on Lie algorithm [6]. The direct method is based on the substitution of the transformation (4) into (2). Imposing the condition $J \neq 0$ leads to a partial differential equation with unknown functions $f$ and $g$, which can be split with respect to some independent variables into the lower order differential equations in $u$ and $t$. The application of the direct method for equation (1) allows us to find two point transformations:

$$
r:(y=t, x=u), \quad r:(n, m, l) \rightarrow(m, n, 3-l), \quad r^{2}=E,
$$

and for $l=0$ :

$s:\left(y=\frac{u}{t}, x=\frac{1}{t}\right), \quad s:(n, m, 0) \rightarrow(-n-m-3, m, 0), \quad s^{2}=E$,

where $E$ is the identity transformation.

\subsection{Bäcklund transformations}

There are many methods of search for Bäcklund transformations such as direct method, RF-pair method and support equations method, which of them are fully discussed in [5]. With application of the Bäcklund transformation for equation 
(1), we obtain the following transformation $g$ :

$$
\begin{aligned}
& g:(n, m, l) \quad \longrightarrow \quad\left(\frac{1}{1-l},-\frac{n}{n+1}, \frac{2 m+1}{m}\right) \\
& \left\{\begin{array} { l } 
{ y _ { x } ^ { \prime } = t ^ { \frac { 1 } { 1 - l } } , } \\
{ y = ( u _ { t } ^ { \prime } ) ^ { - \frac { 1 } { m } } , } \\
{ x = u ^ { \frac { 1 } { n + 1 } } , }
\end{array} \quad \left\{\begin{array}{l}
u_{t}^{\prime}=y^{-m}, \\
u=x^{n+1}, \\
t=\left(y_{x}^{\prime}\right)^{1-l},
\end{array} g^{3}=E=(g r)^{2} .\right.\right.
\end{aligned}
$$

Now by changing the indices of the above expressions assuming the transformed equation as the original and conversely, we obtain a transformation $g^{-1}$ :

$$
\begin{aligned}
& g^{-1}:(n, m, l) \quad \longrightarrow \quad\left(-\frac{m}{m+1}, \frac{1}{l-2}, \frac{n-1}{n}\right) \\
& \left\{\begin{array} { l } 
{ y _ { x } ^ { \prime } = u ^ { \frac { 1 } { 2 - l } } , } \\
{ y = t ^ { \frac { 1 } { m + 1 } } , } \\
{ x = ( u _ { t } ^ { \prime } ) ^ { \frac { 1 } { n } } }
\end{array} \quad \longrightarrow \left\{\begin{array}{l}
u_{t}^{\prime}=x^{n}, \\
u=\left(y_{x}^{\prime}\right)^{2-l}, \\
t=y^{m+1} .
\end{array}\right.\right.
\end{aligned}
$$

\subsection{Solvable equations}

The class of generalized Emden-Fowler equation (1) admits a general group $D_{3}(g, r)$. (For feather details see $\left.[5,6]\right)$

The group $D_{3}(g, r)$, is valid for all values $n, m$ and $l$, except for the singular point $m=-1,0 ; n=-1,0 ; l=1,2$. The group $D_{3}(g, r)$ may be extended smoothly on these points (except for the trivial solvable points $m, n=0$ ) while retaining the group $D_{3}(g, r)$ structure. The technique for constructing the smooth extension is described in [2,5].

Now, by extending the classical Emden-Fowler equations associated to the vector $(1-m, m, 0)$ described in [2,5] and using the operators $g, r$ and $s$ with the consideration of equation (1), we obtain the group $D_{6}(g, r, s)$. This group is valid for every value of $m$ except the singular points $0, \pm 1,2$.

It is necessary to pay attention to the point that using the properties of discrete groups and inevitability of the applied transformations we can reach to the analytic solution of any equation appearing in group $D_{3}(g, r)$, if we can obtain the solution of a single equation by using classical methods. We summarize the selected results from this procedure in Table 1. (see [2]) 


\begin{tabular}{|l|l|}
\hline Differential equation & Analytical solution \\
\hline$y_{x x}^{\prime \prime}=A x^{-4} y^{5}$ & $y(x)=x\left(1+\frac{1}{3} x^{2}\right)^{-\frac{1}{2}}$ \\
$y_{x x}^{\prime \prime}=A x y^{-4}\left(y^{\prime}\right)^{3}$ & $x=y \sin \left(\frac{1}{y}\right)$ \\
$y_{x x}^{\prime \prime}=A x^{-4} y$ & $y(x)=x \sin \left(\frac{1}{x}\right)$ \\
$y_{x x}^{\prime \prime}=A x y^{-\frac{4}{3}}\left(y^{\prime}\right)^{\frac{11}{5}}$ & $y(x)=\left[3\left(x^{-\frac{2}{3}}-1\right)\right]^{-\frac{3}{2}}$ \\
$y_{x x}^{\prime \prime}=A x^{-\frac{5}{6}} y^{-\frac{1}{2}}\left(y^{\prime}\right)^{\frac{5}{4}}$ & $y(x)=\left(1-3^{\frac{3}{5}} x^{\frac{1}{3}}\right)^{3}$ \\
$y_{x x}^{\prime \prime}=A x^{-\frac{1}{2}} y^{-\frac{5}{6}}\left(y^{\prime}\right)^{\frac{7}{4}}$ & $y(x)=3^{-\frac{9}{5}}\left(1-x^{3}\right)^{3}$ \\
$y_{x x}^{\prime \prime}=A x^{-\frac{4}{3}} y\left(y^{\prime}\right)^{\frac{4}{5}}$ & $y(x)=\left[\frac{1}{3}\left(x^{-\frac{2}{3}}+3\right)\right]^{-\frac{3}{2}}$ \\
$y_{x x}^{\prime \prime}=A x^{5} y^{-4}\left(y^{\prime}\right)^{3}$ & $y(x)=\left(x^{-2}-\frac{1}{3}\right)^{-\frac{1}{2}}$ \\
\hline
\end{tabular}

Table 1.

\section{Analysis of the method}

In this section presents analysis of the relation between the exact solutions of classical Emden-Fowler equation and nonlinear equations of heat conduction.

\subsection{The formulation of the problem}

According to [3], we consider the following problem of heat conduction in the spherical coordinates $r, \theta, \phi$ :

$$
\nabla \cdot(K(T) \nabla T)=\frac{\partial T}{\partial t},
$$

with the following initial and boundary conditions:

$$
T(r, 0)=T_{0}, \quad T(1, t)=g(t) .
$$

where $T=T(r, t)$ is the temperature, $K$ the heat transfer coefficient, and $t$ the time. Assume that

$$
K=k_{0} T^{\frac{5}{2}}
$$

where $k_{0}$ is constant.

Equation (5) can be written as

$$
\frac{\partial K}{\partial r} \widehat{r} \cdot \frac{\partial T}{\partial r} \widehat{r}+\frac{K}{r^{2}} \frac{\partial}{\partial r}\left(r^{2} \frac{\partial T}{\partial r}\right)=\frac{\partial T}{\partial t} .
$$


By substituting:

$$
\frac{\partial K}{\partial r}=\frac{5 k_{0}}{2} T^{\frac{3}{2}} \frac{\partial T}{\partial r},
$$

into (6), we obtain the following equation:

$$
\frac{5 k_{0}}{2} T^{\frac{3}{2}}\left(\frac{\partial T}{\partial r}\right)^{2}+\frac{2 k_{0}}{r} T^{\frac{5}{2}} \frac{\partial T}{\partial r}+k_{0} T^{\frac{5}{2}} \frac{\partial^{2} T}{\partial r^{2}}=\frac{\partial T}{\partial t} .
$$

Application of the separation of variables:

$$
T(r, t)=R(r) P(t),
$$

gives:

$$
\frac{\partial T}{\partial t}=R P_{t}^{\prime}, \quad \frac{\partial T}{\partial r}=P R_{r}^{\prime}, \quad \frac{\partial^{2} T}{\partial r^{2}}=P R_{r r}^{\prime \prime},
$$

and hence equation (7) reduces to:

$$
\frac{5 k_{0}}{2} R^{\frac{1}{2}} R_{r}^{\prime 2}+2 k_{0} r^{-1} R^{\frac{3}{2}} R_{r}^{\prime}+k_{0} R^{\frac{3}{2}} R_{r r}^{\prime \prime}=P^{-\frac{7}{2}} P_{t}^{\prime}=-\lambda^{2},
$$

where $\lambda$ is the separation parameter. As a result of this decomposition, we obtain the following defining system:

$$
\left\{\begin{array}{l}
P^{-\frac{7}{2}} P_{t}^{\prime}=-\lambda^{2}, \\
\frac{5 k_{0}}{2} R^{\frac{1}{2}} R_{r}^{\prime 2}+2 k_{0} r^{-1} R^{\frac{3}{2}} R_{r}^{\prime}+k_{0} R^{\frac{3}{2}} R_{r r}^{\prime \prime}=-\lambda^{2} .
\end{array}\right.
$$

Note that the first equation of this system yields the function $P(t)$, in the following form:

$$
P(t)=\left(\frac{5}{2} \lambda^{2} t+c_{1}\right)^{-\frac{2}{5}}
$$

where $c_{1}$ is a constant.

We shall now apply the following $R F$-pair operations, as a consequence of which the second equation of the system (10) is transformed to the classical Emden-Fowler equation. Having applied the operation:

$$
F U: r=e^{s}, \quad R=u e^{k s},
$$


where

$$
\begin{aligned}
R_{r}^{\prime} & =e^{s(k-1)}\left(u_{s}^{\prime}+k u\right), \\
R_{r r}^{\prime \prime} & =e^{s(k-2)}\left(u_{s s}^{\prime \prime}+(2 k-1) u_{s}^{\prime}+k(k-1) u\right),
\end{aligned}
$$

on the second equation of the system (10), we have the following equation:

$$
e^{s\left(\frac{5 k}{2}-2\right)}\left(u_{s s}^{\prime \prime}+\frac{5}{2} u^{-1} u_{s}^{\prime 2}+(7 k+1) u_{s}^{\prime}+\left(\frac{7 k^{2}}{2}+k\right) u+\frac{\lambda^{2}}{k_{0}} u^{-\frac{3}{2}}\right)=0,
$$

and if we set $k=\frac{4}{5}$, then equation (12) reduces to the autonomous equation

$$
u_{s s}^{\prime \prime}+\frac{5}{2} u^{-1} u_{s}^{\prime 2}+\frac{33}{5} u_{s}^{\prime}+\frac{76}{25} u+\frac{\lambda^{2}}{k_{0}} u^{-\frac{3}{2}}=0 .
$$

Now, by using the raising order operation $F X: u_{s}^{\prime}=w(u), \quad u_{s s}^{\prime \prime}=w w_{u}^{\prime}$, equation (13) reduces to the general Abel equation to the following form:

$$
w w_{u}^{\prime}=-\frac{3}{2} u^{-1} w^{2}-\frac{33}{5} w-\frac{76}{25} u-\frac{\lambda^{2}}{k_{0}} u^{-\frac{3}{2}},
$$

where the general coefficients of this Abel equation according to the definition 3 , is as follows:

$$
\begin{gathered}
\phi_{0}(u)=0, \quad \phi_{1}(u)=1, \\
\psi_{0}(u)=-\frac{\lambda^{2}}{k_{0}} u^{-\frac{3}{2}}-\frac{76}{25} u, \quad \psi_{1}(u)=-\frac{33}{5}, \quad \psi_{2}(u)=-\frac{5}{2} u^{-1} .
\end{gathered}
$$

In this position, we consider the following transformations:

$$
E=\exp \left(-\int \frac{\psi_{2}(u)}{\phi_{1}(u)} d u\right)=u^{\frac{5}{2}}, \quad v=\left(\frac{\phi_{0}(u)}{\phi_{1}(u)}+w\right) E=u^{\frac{5}{2}} w
$$

and define:

$$
\begin{aligned}
& F_{0}(u)=\left(\frac{\psi_{0}}{\phi_{1}}-\frac{\phi_{0} \psi_{1}}{\phi_{1}^{2}}+\frac{\phi_{0}^{2} \psi_{2}}{\phi_{1}^{3}}\right) E^{2}-\frac{\lambda^{2}}{k_{0}} u^{\frac{7}{2}}-\frac{76}{25} u^{6}, \\
& F_{1}(u)=\left[\frac{d}{d u}\left(\frac{\phi_{0}}{\phi_{1}}\right)+\frac{\psi_{1}}{\phi_{1}}-2 \frac{\phi_{0} \psi_{2}}{\phi_{1}^{2}}\right] E-\frac{33}{5} u^{\frac{5}{2}} .
\end{aligned}
$$


By applying the transformation (15) on the equation (14), we obtain:

$$
v v_{u}^{\prime}=-\frac{33}{5} u^{\frac{5}{2}} v-\frac{\lambda^{2}}{k_{0}} u^{\frac{7}{2}}-\frac{76}{25} u^{6} .
$$

The equation (16) by using the transformations:

$$
\tau=\tau(u)=\int F_{1}(u) d u=-\frac{33}{5} \int u^{\frac{5}{2}} d u=-\frac{66}{35} u^{\frac{7}{2}},
$$

and

$$
\varphi(u)=\frac{F_{0}(u)}{F_{1}(u)}=\frac{76}{165} u^{\frac{7}{2}}+\frac{5 \lambda^{2}}{33 k_{0}} u
$$

reduce to

$$
v v_{\tau}^{\prime}-v=\frac{5 \lambda^{2}}{33 k_{0}} \tau+\frac{76}{165} \tau^{\frac{7}{2}}
$$

where

$$
\tau=-\frac{66}{35} u^{\frac{7}{2}}
$$

Now, by introducing the new constants $n$ and $A$ in the forms:

$$
\frac{5 \lambda^{2}}{33 k_{0}}=-\frac{(n+2)\left(n+\frac{9}{2}\right)}{\left(2(n+2)+\frac{5}{2}\right)^{2}}, \quad \frac{76}{165}=A\left(\frac{5}{4(n+2)+5}\right)^{2},
$$

and using the transformation:

$$
f=A \tau^{\frac{5}{2}}, \quad z=\frac{5+4(n+2)}{5} \frac{v}{\tau}-\frac{2(n+2)}{5},
$$

equation (17) reduced to the equation:

$$
\left(z-z^{2}+f\right) f_{z}^{\prime}=\left(\frac{5}{2} z+n+2\right) f .
$$

(For details see [8]).

Finally, through the substitution:

$$
f=A x^{n+2} y^{\frac{5}{2}}, \quad z=\frac{x}{y} y_{x}^{\prime},
$$

the equation (21) is transformed to the classical Emden-Fowler equation:

$$
y_{x x}^{\prime \prime}=A x^{n} y^{\frac{7}{2}},
$$


where $A$ is an insignificant parameter, $A$ and $n$ depends on $\lambda$ and $\lambda$ has been defined in (9) as a separation parameter. Note that $n$ is also a constant depending on the initial and boundary conditions. For some values of $n$, this equation is integrable as a consequence of which, we have been able to construct some solution for the nonlinear heat conduction problem.

\subsection{Exact solutions of the original equation}

In this section, our aim is to obtain the exact solutions of the second equation of system (10), using the effect of inverse of the $R F$-pair operators on the exact solutions of the Emden-Fowler equation (23). For this purpose, in equation (23) let $n=-\frac{13}{2}$, then this equation is reduced to the following equation:

$$
y_{x x}^{\prime \prime}=A x^{-\frac{13}{2}} y^{\frac{7}{2}}
$$

with parametric solution as:

$$
x=\alpha C_{1}^{* l} N\left(\theta, C_{2}^{*}\right)=\alpha C_{1}^{* l} E_{\frac{7}{2}}^{-1}, \quad y=\beta C_{1}^{* h} M\left(\theta, C_{2}^{*}\right)=\beta C_{1}^{* h} \theta E_{\frac{7}{2}}^{-1},
$$

where

$$
\begin{gathered}
A= \pm \frac{9}{4} \alpha^{\frac{9}{4}} \beta^{-\frac{5}{2}}, \quad h=\frac{9}{2}, \quad l=\frac{5}{2}, \\
E_{\frac{7}{2}}=\int\left(1 \pm \theta^{\frac{9}{2}}\right)^{-\frac{1}{2}} d \theta+C_{2}^{*}, \quad N=E_{\frac{7}{2}}^{-1}, \quad M=\theta E_{\frac{7}{2}}^{-1},
\end{gathered}
$$

and $C_{1}^{*}, C_{2}^{*}, \alpha$ and $\beta$ are constants and $A=f(\alpha, \beta)$ is an insignificant parameter which is fully discussed in [6], pp. 254-255.

By using the parametric solutions (20), we have

$$
y_{x}^{\prime}=\frac{d y}{d x}=\frac{\beta}{\alpha} C_{1}^{*(h-l)}\left(\theta-\frac{E_{\frac{7}{2}}}{\frac{d}{d \theta} E_{\frac{7}{2}}}\right),
$$

and substituting (24) and (251) in the transformation (22), we obtain the exact solution of equation (21) in the following form:

$$
f=A \alpha^{-\frac{9}{2}} \beta^{\frac{5}{2}} C_{1}^{*\left(\frac{5 h-9 l}{5}\right)} \theta^{\frac{5}{2}} E_{\frac{7}{2}}^{2}, \quad z=1-\frac{E_{\frac{7}{2}}}{\theta \frac{d}{d \theta}\left(E_{\frac{7}{2}}\right)} .
$$


From equation (26) and (20), we obtain $\tau$ as:

$$
\tau=\alpha^{-\frac{9}{5}} \beta C_{1}^{*\left(\frac{5 h-9 l}{5}\right)} \theta E_{\frac{7}{2}}^{\frac{4}{5}},
$$

and also using the relation (19), we reformulate the expression for $z$ in (20) as follows:

$$
z=\frac{662 k_{0}}{5 \lambda^{2}} \frac{18}{65} \frac{v}{\tau}+\frac{9}{5}
$$

Now, by substituting transformations (26) and (27) into (28), we obtain the parametric solution of the equation (17) in the following form:

$$
v=B_{0} \alpha^{-\frac{9}{5}} \beta C_{1}^{*\left(\frac{5 h-9 l}{5}\right)} \lambda^{2} \theta\left(-\frac{4}{5}-\frac{E_{\frac{7}{2}}}{\theta \frac{d}{d \theta} E_{\frac{7}{2}}}\right) E_{\frac{7}{2}}^{\frac{4}{5}},
$$

where

$$
B_{0}=\frac{325}{688 k_{0}} .
$$

On the other hand, by using (27), we have:

$$
\theta E_{\frac{7}{2}}^{\frac{4}{5}}=\alpha^{\frac{9}{5}} \beta^{-1} C_{1}^{*\left(\frac{5 h-9 l}{5}\right)} \tau
$$

and substituting (30) in (29), the equation (29) is reduced to the following equation:

$$
v(\tau)=-B_{0} \lambda^{2} \tau\left(\frac{4}{5}+\frac{D_{2}(\tau)}{\tau D_{1}(\tau)}\right)
$$

where

$$
\begin{aligned}
& D_{1}(\tau)=\left.\frac{d E_{\frac{7}{2}}}{d \theta}\right|_{\theta=\alpha^{\frac{9}{5}} \beta^{-1} C_{1}^{*\left(\frac{5 h-9 l}{5}\right)} E_{\frac{7}{2}}^{-\frac{4}{5}} \tau}, \\
& D_{2}(\tau)=\left.\alpha^{-\frac{9}{5}} \beta C_{1}^{*\left(\frac{5 h-9 l}{5}\right)} E_{\frac{7}{2}}\right|_{\theta=\alpha^{\frac{9}{5}} \beta^{-1} C_{1}^{*\left(\frac{5 h-9 l}{5}\right)} E_{\frac{7}{2}}^{-\frac{4}{5}} \tau} .
\end{aligned}
$$

Now, the solution of (16) by using the relations (18) and (31) can be obtained as:

$$
v(u)=-B_{0} \lambda^{2} u\left(\frac{4}{5}+\frac{D_{2}(u)}{u D_{1}(u)}\right),
$$


where

$$
u=\left(-\frac{7}{14 k+2}\right)^{\frac{2}{7}} \tau^{\frac{2}{7}}
$$

Also, by replacing (15) into (32), we obtain the following solution for equation (14):

$$
w(u)=-B_{0} \lambda^{2} u^{-\frac{3}{2}}\left(\frac{4}{5}+\frac{D_{2}(u)}{u D_{1}(u)}\right) .
$$

Applying the operator

$$
F X^{-1}: s=\int \frac{d u}{w(u)}
$$

on the equation (33), yields the exact solution of the equation (13) in the following form:

$$
s=-\frac{1}{B_{0} \lambda^{2}} \int \frac{u^{\frac{3}{2}}}{\frac{4}{5}+\frac{D_{2}(u)}{u D_{1}(u)}} d u .
$$

Now, if we assume that this equation is integrable, then we set:

$$
s=\frac{1}{B_{0} \lambda^{2}} F(u)
$$

where

$$
F(u)=-\int \frac{u^{\frac{3}{2}}}{\frac{4}{5}+\frac{D_{2}(u)}{u D_{1}(u)}} d u
$$

Hence, by using the inverse operator $F U: r=e^{s}, R(r)=u r^{k}$ in equation (34), this equation reduces to the exact solution of the second equation of system (10), which is as follows:

$$
R(r)=u e^{k \frac{1}{B_{0} \lambda^{2}} F(u)},
$$

where $u$ is a function of $r$. Finally, by substituting (11) and (35) in (8), we get the exact solution of equation (7) in spherical coordinate as follows:

$$
T(r, t)=R(r) P(t)=\left(u e^{k \frac{1}{B_{0} \lambda^{2}} F(u)}\right)\left(a \lambda^{2} t+c_{1}\right)^{-\frac{2}{5}} .
$$

Due to the symmetry of the problem with respect to $\theta$ and $\phi$, we may set $\phi=\frac{\pi}{2}$, $\theta=0$ and obtain the same initial and boundary condition as $T(r, 0)=T_{0}$, $T(1, t)=g(t)$. 


\section{Conclusion}

By introducing the some $R F$-pair operation and series of transformations beside $D G T$, the nonlinear heat conduction problem with variable transfer coefficient in applied physics can be transformed into the classical Emden-Fowler equation which maybe integrated using classical methods. Then, by using the inverse of this operations and transformations, the exact solution of Emden-Fowler equation reduced to the exact solution of the nonlinear heat conduction problem. This approach shows that, under the $R F$-pair operations and transformations, the solution of transformed equation can be converted into the solution of the reference equation.

\section{REFERENCES}

[1] P. Darania and M. Hadizadeh, On the RF-pair operations for the exact solution of some classes of nonlinear Volterra integral equations, Mathematical Problem in Engineering, 1(2006), $1-11$.

[2] M. Hadizadeh, A.R. Zokayi and P. Darania, On the Discrete Group Analysis for Solving Some Classes of Emden-Fowler Equations, Applied Mathematics Research Express, 5(2004), 169-178.

[3] P. Darania and A. Ebadian, Discrete Group Methods for Nonlinear Heat Equations, Kyungpook Math J. 46 (2006), 329-336.

[4] P. Darania, A. Ebadian and A.V. Oskoi, Linearization method for solving nonlinear integral equations, Mathematical Problem in Engineering, 1(2006), 1-11.

[5] V.F. Zaitsev and A.D. Polyanin, Discrete-Group Methods for Integrating Equations of Nonlinear Mechanics, CRC Press, California, (1994).

[6] V.F. Zaitsev, On discrete-group analysis of ordinary differential equations, Soviet Math. Dok1., 37(1988), 403-406.

[7] G. Böhme, Non-Newtonian Fluid Mechanics, North-Holland Series in Applied Mathematics and Mechanics, (31), Amsterdam, (1987).

[8] J.J. Duderstadt and G.A. Moses, Inertial Confinement Fusion, John Wiley and Sons, New York, (1982).

[9] A.J. Kemp, M.M. Basko and J. Meyer-ter-Vehn, Ignition conditions for magnetically insulated tamped ICF targets in cylindrical geometry, Nucl. Fusion, 41(2001), 235-241.

[10] L.V. Ovsiannikov, Group Analysis of Differential Equations, Academic Press, New York, (1982). 